\title{
Ideal docente y planificación real: un estudio de caso en formación inicial
}

\author{
Nuria Abal Alonso, Eduardo José Fuentes Abeledo, Pablo César Muñoz Carril \\ Dpto. de Didáctica y Organización Escolar, Universidad de Santiago de Compostela
}

\begin{abstract}
Resumen
Esta comunicación procede del trabajo en el marco de un proyecto de investigación, de enfoque tanto cuantitativo como cualitativo, que tiene por objetivo básico profundizar en el conocimiento de los procesos y resultados de la formación de maestros y maestras de Educación Infantil en centros universitarios gallegos. En esta ocasión presentamos hallazgos en torno al ámbito de la planificación docente, procedentes de un estudio de caso desarrollado con una aprendiz de maestra, a partir del análisis de los datos recogidos tanto antes, como durante y después de su primera experiencia de prácticas en un centro escolar durante el segundo curso del plan de estudios.
\end{abstract}

Palabras clave: practicum, educación infantil, planificación, formación inicial de maestros, estudio de caso.

\section{Contexto, objetivos y metodología}

En el proyecto de investigación no subvencionado en el que estamos inmersos y que integra los enfoques cuantitativo y cualitativo, pretendemos profundizar en el conocimiento de los procesos y resultados de la formación inicial de maestros y maestras de Educación Infantil en el contexto gallego. Esta comunicación, que se complementa con otra presentada a este mismo Congreso, responde a una de las líneas de trabajo en la que pretendemos indagar en torno a la propuesta curricular de la Universidad de Santiago de Compostela para la formación de maestras y maestros de Educación Infantil (véase una presentación y análisis de la misma en Fuentes Abeledo, 2009).

Entre nuestros objetivos destacamos el de identificar, describir y analizar las concepciones, conocimientos, dilemas y competencias -y su evolución- que mantienen los futuros profesores de Educación Infantil en relación con la tarea docente en esta etapa, prestando especial atención al impacto de las experiencias de prácticas en los centros escolares y a los elementos que influyen en la configuración de la perspectiva de los futuros profesores y profesoras de esta etapa.

Hemos optado por el estudio de caso (Simons, 2011) como estrategia metodológica. En esta aportación, y en un marco conceptual de índole hermenéutica, fenomenológica e interpretativa, nos servimos de los datos recogidos tanto antes, como durante y después de la primera experiencia de prácticas situada en el primer cuatrimestre del segundo curso de la carrera. La información recogida procede de múltiples fuentes: cuaderno de reflexión y otros documentos de una materia teórico-práctica ubicada en el mismo cuatrimestre que el Practicum I (Documento 1) y otros Documentos (D2 y D3) producidos por Isabel (nombre supuesto de la futura docente que participa en la investigación) a raíz de su experiencia de prácticas (Practicum I) en un aula del segundo ciclo de Educación Infantil. Aunque los datos analizados proceden básicamente de las fuentes citadas, también hemos revisado los originados en las entrevistas, grupos de discusión y seminarios de trabajo en los que ha participado Isabel.

En este estudio cualitativo para analizar los datos realizamos varias lecturas exploratorias para pasar a continuación a desarrollar un proceso de codificación y clasificación que ha permitido penetrar en el significado de las producciones de Isabel, emergiendo un conjunto de categorías. Son numerosas las categorías que han ido surgiendo (por ejemplo: finalidades de la Educación Infantil, aprendizaje, niño, maestro, clima de aula, metodología, institución, currículo, modelo didáctico, etc.) y de subcategorías para cada una de las mismas. Este proceso nos han permitido ordenar las concepciones (diferenciándolas de descripciones neutras, valoraciones positivas o problemas), y dotar de sentido al conjunto para ir desvelando la "perspectiva" de "Isabel", siempre siguiendo la sabia recomendación de Patton (1980) para quien el análisis cualitativo consiste fundamentalmente en "hacer lo más que se pueda para darle sentido -descifrar las cosas-" (p. 345).

En esta comunicación presentamos una primera aproximación a las concepciones centradas en una de las tareas propias del profesorado: la planificación.

\section{Planificación y compromiso profesional docente: la perspectiva de Isabel}

\section{Unas concepciones claras, con articulación interna y argumentación explícita de diversa procedencia}

La perspectiva de Isabel, antes de incorporarse a su primera experiencia de prácticas en centros, en torno a la relevancia de la planificación docente más allá de exigencias de la Administración, y a su importancia, se pone de manifiesto en múltiples declaraciones, así como los argumentos que avalan tal convicción. Es más, no concibe la tarea docente sin disponer y usar herramientas en relación con la planificación. En palabras de Isabel, "la planificación es algo fundamental que sirve de soporte al maestro" (D1, 8/13), destacando varias razones que expone en su cuaderno de reflexión en diferentes textos y fechas. 
Los argumentos más destacados sitúan como eje al niño. Según ella si se prescinde de la planificación "el más perjudicado es el alumno" (D1, 8/13) porque, por ejemplo, puede que no se aborden todos los objetivos de "formación y educación" (D1, 8/13), resintiéndose entonces su "formación integral". Este planteamiento queda muy claro cuando, en los textos que hemos analizado, critica con fuerza la actuación de una docente en el contexto de diversas actividades de estudio del caso concreto de una profesora en el marco de una de las materias teórico-prácticas de la titulación. Todo ello en coherencia con su concepción general de la Educación Infantil. Además, comenta Isabel, contar con una planificación a la hora de afrontar los avatares de la vida del aula, conlleva reflexionar a fondo en la fase preactiva de la enseñanza, y ello "facilita la labor" del docente en su relación con el alumnado (D1, 8/15).

Por otra parte, conoce que no siempre se dedica el tiempo oportuno a esa fase preactiva, y critica que algunos puedan considerarlo "una formalidad que presentar a la institución" (D1, 8/14), con el peligro sobre todo de que aspectos importantes del desarrollo del niño como persona puedan ser "ignorados y sobreseídos" (D1, 9/4). Esta futura docente añade una razón más que avala su posicionamiento claro a favor de una planificación escrita, bien pensada: "es completamente necesario tener una idea completa acerca del conjunto del proceso" (D1, 8/14). Su ausencia, en su opinión, desvelaría "falta de compromiso y responsabilidad" del profesorado (D1, 8/14) y ello va en menoscabo de la realización auténtica del profesional de la docencia.

Por tanto, construye un ideal de docente responsable y comprometido en coherencia con su concepción de la Educación Infantil, exigencia que conlleva una especial atención a tareas como la planificación, desde la visión de un profesor que actúe como facilitador, promotor e impulsor del progreso infantil" (D1, 9/4) y que no se olvida del "perfeccionamiento de la personalidad" de cada niño. (D1, 9/4).

El "compromiso" al que se refiere no lo liga únicamente a un contrato legal del docente, sino que se relaciona directamente con un compromiso moral que surge en relación con la máxima atención y cuidado de los pequeños, con su derecho a una "educación integral" (D1, 29/2), atenta a "aspectos vinculados a la constitución y perfeccionamiento de la personalidad" (D1, 9/4). Una apuesta que, defiende Isabel, ha de estar muy atenta a la diversidad, al desarrollo de todas las potencialidades, de todas las dimensiones de la persona, pensando siempre en todos los alumnos y alumnas, algo que ha de propiciarse desde la fase de planificación. Por otra parte, Isabel declara con nitidez que en la base de la actuación docente que considera adecuada ha de anidar un fuerte compromiso personal: "Considero que el papel de los maestros depende exclusivamente de nosotros y del compromiso que tengamos con los alumnos y con la profesión" (D1, 6/9).

Pero en relación con este planteamiento, desde la perspectiva de Isabel, ¿qué tipo de planificación es capaz de responder a este reto?. Para ella en la fase preactiva resulta imprescindible que el profesor reflexione sobre el contexto y grupo concreto de alumnos: "es preciso reflexionar sobre cada aula, realizando una programación que responda a la lógica de lo contextual, de lo situacional" (D1, 12/6). Por ello reclama que, en todo caso, cualquier intento de "implementar" en el aula propuestas curriculares externas a la misma, se base "en la adaptación" (D1, 12/6), y siempre pensando que cualquier propuesta definida debe ser flexible, atenta en su desarrollo en el aula a las múltiples circunstancias y requerimientos que puedan producirse sobre la marcha. En definitiva, para esta futura profesora la planificación significa fundamentalmente reflexionar.

\section{Representación del trabajo docente y tareas visibles e invisibles en las prácticas escolares: la función de planificación}

El trabajo docente no se circunscribe únicamente al mantenimiento de relaciones con los niños con afán de propiciar su aprendizaje y desarrollo. Entre las funciones del profesorado, como destaca Isabel, se encuentra también la planificación. En las aulas universitarias durante la formación inicial y antes de incorporarse a su primera estancia en los centros escolares, ha tenido acceso a conocimientos diversos que, de una u otra forma, han podido influir sobre las concepciones declaradas que mantiene sobre esta cuestión, algunas de las cuales hemos presentado en el apartado anterior. Queremos subrayar su visión de la planificación como "reflexión" preactiva del docente que posibilita la contextualización y adaptación.

Esa representación de una de las funciones docentes establecidas normativamente, $y$ que se liga a determinadas competencias del profesorado, va a ser explorada por Isabel desde el comienzo de su primera experiencia de prácticas desarrollada en el segundo ciclo de Educación Infantil con una docente con experiencia. La Ley Orgánica de Educación, se refiere a la misma con el término "programación" (diferenciándola de "enseñanza" y "evaluación”), por lo que también su tutora usará esta expresión. Una función que conlleva el despliegue de actividades que el alumnado en prácticas no siempre puede observar directamente puesto que, en relación con la misma, las actividades no suelen desarrollarse con los alumnos, al igual que otras, como, por ejemplo, el encuentro con los padres o el trabajo de coordinación con otros profesores. Por tanto, según nuestra experiencia trabajando con alumnado en prácticas, en no pocas ocasiones se convierten en actividades del tutor o tutora "invisibles" para los docentes en formación inicial, y sin delimitaciones fijadas estrictamente en su proceso de desarrollo, a diferencia, por ejemplo, de las tareas interactivas de enseñanza para las que se fija su duración por convención.

En su estancia en el centro, Isabel no sólo ha observado aspectos del desempeño de diversas funciones del profesorado, especialmente de su tutora, sino también ha explorado el significado que otorga al acontecer de la vida del aula y al desarrollo de diversas tareas a través de conversaciones esporádicas y 
entrevistas, tanto formales como informarles, sirviéndose también del análisis de documentos. Es decir, ha accedido a lo que sucede en clase pero también ha indagado en torno a las razones y argumentos de por qué, desde la perspectiva de su tutora, suceden algunas cosas en la vida del aula llegando a conclusiones sobre la forma particular que tiene de pensar y ejercer la docencia dicha profesora y se fija, por ejemplo, en el ámbito de la "programación" reflexionado sobre las consecuencias que ésta tiene sobre el alumnado en múltiples dimensiones.

a) Programación "burocrática" y "real".

Isabel describe cómo afronta su tutora la labor de planificar o programar lo que se va hacer en el aula con matices de desilusión y cuestionamiento al comprobar cómo justifica la existencia de documentación de planificación como una necesidad derivada de la exigencia burocrática y administrativa, más que para atender a las funciones y necesidades que esta futura docente considera pertinentes y que hemos resumido anteriormente.

Por tanto, como la propia tutora le manifiesta en las entrevistas, los documentos escritos de la planificación que se entregan a la dirección y que se envían a la inspección responden a una necesidad "burocrática", a una exigencia reglamentaria para el profesorado con unos formatos determinados, y que ella pone a disposición del cuerpo específico de la administración educativa que vela por su cumplimiento.

Esta planificación es denominada por la tutora como "programación global" (D2, 2/7 y 3/1), un documento que ha elaborado con otras compañeras y que resulta una garantía para hacer frente a las exigencias administrativas de control externo. Sin embargo, la propia tutora comenta que luego dispone de otra planificación "diaria", "en la vida diaria" que identifica con la propuesta curricular contenida en el "Libro del Profesor" que se corresponde con el material del que se sirve para el acontecer cotidiano en el aula (fundamentalmente los bloques de "fichas" del material publicado por una editorial y que aparece en la "Guía del Profesor"). Esta planificación, ligada al trabajo del día a día, concreta actividades y experiencias a realizar con el alumnado, que pueden "modificarse" -o surgir nuevas actividades-. Puede "cambiarse", como explica la tutora, según el acontecer y "problemas" de la vida del aula (D3, 2/8). El núcleo fundamental de esta propuesta curricular se vehicula a través de las "fichas" (D3, 3/9), que son consideradas por la tutora un material "muy importante" puesto que, según manifiesta, "ayudan a trabajar con el niño para conseguir un fin (...), facilitando el trabajo con los alumnos" (D3, 3/9).

La "'programación global" a la que se refiere la tutora, es denominada por Isabel "planificación ficticia" (D3, 5/5), respetando el sentido y finalidades que le adjudica la propia docente. La planificación "real" se correspondería pues, con el "currículo editado" que se convierte en la herramienta fundamental para el desarrollo de las clases. Desde la visión de Isabel, la "experiencia docente" (D2, 58/4) le permite a su tutora, consultando brevemente la "Guía del Profesor", afrontar la labor cotidiana realizando actividades "de temática vinculada con las fichas" (D2, 58/4) de una forma que Isabel califica como "espontánea e improvisada" (D2, 58/4).

b) Presencias y ausencias curriculares.

A través de la observación de la marcha cotidiana de las clases, del análisis de documentación y materiales curriculares usados habitualmente y de las conversaciones con la tutora, Isabel va accediendo no sólo a la plasmación del currículo en acción en el aula, sino también a algunos de los supuestos declarados de su tutora, y la relación con las materiales curriculares utilizados, centrándose en argumentaciones sobre el fuerte énfasis puesto en todas aquellas actividades que tengan que ver con la lecto-escritura y aprendizaje de algunas nociones matemáticas. Así, la numeración, conteo, abecedario, grafomotricidad, para la tutora son "imprescindibles" puesto, que según comenta a Isabel, "las ciencias y las letras son los campos del saber por antonomasia" (D3, 3/7). Este planteamiento nos lleva a pensar en las dificultades de desarrollar currículos integrados y el enfoque de proyectos puesto que, como ha argumentado Apple (1986), la escuela opta habitualmente por "maximizar la producción de conocimiento de status elevado" (p. 38).

Estas y otras manifestaciones y observaciones, conducen a Isabel a identificar concepciones fundamentales de su tutora en torno al sentido y finalidades de la Educación Infantil, que también se hacen explícitas al declarar que: "en estas edades tan tempranas es necesario que las maestras de Educación Infantil, visualicemos nuestra importancia en las etapas posteriores" (D3, 3/7).

Los múltiples ejemplos que recoge de diversos momentos de actividades a lo largo de los días de estancia en el aula desvelan a lo que se presta atención prioritaria en tiempo de clase, y lo que se evalúa del quehacer de los pequeños. No le queda la menor duda acerca de la perspectiva que defiende su tutora y que, además, la misma profesora expresa con claridad: evitar que los pequeños "tengan problemas en cursos superiores" (D3, 3/3), que no "tengan dificultades más adelante" (D3, 3/5). Para Isabel, la tutora defiende "concepciones vinculadas a aspectos tradicionales" (D3, 4/18), centrando el trabajo sobre los contenidos citados y prestando escasa atención a otros contenidos menos "académicos", como por ejemplo los artísticos o ligados al desarrollo adecuado en los ámbitos afectivo, emocional y social.

c) Impactos sobre el alumnado y la profesionalización docente.

El análisis del conjunto de los datos recogidos permite desvelar cómo interpreta y valora Isabel la presencia en la realidad de la escuela de lo que ella misma denomina "planificación ficticia" y "planificación real", así como lo que hemos rotulado como "presencias y ausencias curriculares", a la luz de la lente de las propias concepciones que ha ido construyendo.

Así, la desconexión entre la "planificación ficticia" y la "planificación real" que desde su punto de vista gobierna la vida del aula, es un impedimento relevante para una adecuada adaptación del currículo oficial al contexto y peculiaridades del alumnado. Este 
planteamiento no responde a las concepciones que ella defiende y que hemos descrita en párrafos anteriores. No identifica la presencia de una planificación, que "responda a la lógica de lo contextual, de lo situacional" (D1, 12/6). Por tanto, esta laguna propicia, por ejemplo, dificultades considerables para que la vida del aula responda a principios que considera fundamentales, como el de "individualización" (D3, 5/5) o el de "socialización" adecuada de los pequeños, o en el desarrollo de una evaluación más atenta "a lo situacional, concreto e inmerso en la realidad del aula" (D3, 6/3), tanto individual como grupal (D3, 6/3 y 7/1).

Por otra parte, las "ausencias curriculares" inciden negativamente sobre aspectos que entiende nucleares en su concepción de la Educación Infantil, como la relevancia del juego en la dinámica cotidiana de la vida del aula, y la necesidad de atender a "temáticas orécticoexpresivas" (D3, 6/1).

Isabel, como hemos visto, defiende con convicción un rol docente con perfil de profesor reflexivo. Desde su perspectiva, la reflexión en la fase "preactiva" de la enseñanza resulta fundamental pues posibilita la “contextualización" y "adaptación" del currículo. Las decisiones de orden curricular han de ser asumidas por los docentes en respuesta a su compromiso profesional. Decisiones que permiten clarificar las intenciones, los principios que han de regular la práctica, los contenidos y su secuencia, así como las estrategias, actividades, materiales y concreciones de evaluación. El análisis de sus manifestaciones previas a las prácticas desvelan ideas claras al respecto. Los documentos analizados en relación con su experiencia en las prácticas también lo evidencian. Isabel tiene muy claro que plasmar por escrito esas decisiones de forma autónoma y reflexiva por parte de cada profesor, y también en colaboración con otros colegas, resulta un aspecto clave de la función docente y que ello exige desarrollar competencias curriculares sirviéndose de las diferentes "fuentes" del currículo. Sobre esta última cuestión resultan muy significativas declaraciones y valoraciones que manifiesta, por ejemplo en relación con lo que observa en la vida del aula y principios como los citados anteriormente de "individualización" y "socialización". Así, por ejemplo, en relación con el primero y la "fuente psicológica" comenta los problemas que pueden derivarse de la falta de atención a los diferentes "ritmos de aprendizaje" de los niños, o de la adopción de una "perspectiva logocéntrica" (D3, 5/5) en la selección y tratamiento de los contenidos en Educación Infantil.

\section{Observaciones finales}

\section{La importancia de comprender las culturas de los agentes educativos: tareas visibles $e$ invisibles $y$ lógicas explicativas}

En el contexto curricular de formación inicial en que transcurre la trayectoria de desarrollo de Isabel, los aprendices de profesores como ella pasan en tres momentos diferentes de la carrera por los centros escolares. La primera experiencia de prácticas a la que nos hemos referido, de unas cuatro semanas de duración, permite una aproximación al conocimiento de algunas de las funciones y tareas docentes y de la vida del aula, permitiendo percibir algunos de los problemas de las realidades de la clase.

Una mínima comprensión de lo que acontece en el aula, exige, en nuestra opinión, acceder a la perspectiva del docente titular para establecer relaciones entre sus acciones, su pensamiento y el contexto, no sólo acceder a datos a través de lo que es visible directamente. Comprender las dinámicas de acción de planificación y relacionarlas con el "compromiso" del docente con su trabajo (compromiso deseable que Isabel describe con claridad antes de su experiencia), requiere atender a elementos de carácter personal (situaciones vitales, relaciones con otros o la salud, por ejemplo), profesional (saberes teóricos, competencias, experiencias, por ejemplo) e institucional (nivel de colaboración entre profesionales en la organización escolar, existencia de un proyecto institucional compartido, por ejemplo).

Los aspectos citados inciden en el nivel de "compromiso" docente como comentan Day (2006) y Hargreaves y Fullan (2014). Un compromiso en el desempeño profesional en relación con cualquiera de las funciones y tareas, como la de planificación, y las peculiares formas que adopta en procesos y resultados requiere para su comprensión, y sólida interpretación, atender a estas variables y situarlas también en relación con tradiciones en el seno de la profesión considerando los límites, condiciones del puesto de trabajo, costumbres, presiones y códigos hegemónicos. Ello facilita que quien observa lo que acontece en el aula enriquezca sus comentarios yendo incluso más allá de las pistas que ofrezca un ideal construido, en buena parte, en un escenario. En esta línea, comprender los casos concretos y compromisos peculiares de personas particulares, requiere dotarse de conceptos potentes, como el de "cultura escolar", que permitan aventurarse, bien pertrechado, en la compleja tarea de desentrañar lo que sucede en la vida de las aulas y comprender las dificultades de instaurar cambios en la línea de concepciones expuestas como las que defiende Isabel, construidas con influencias notables de las materias teórico-prácticas que ha cursado antes de su inmersión en las primeras prácticas. Una "cultura escolar" que cabe entender como "un conjunto de teorías, de ideas, principios, normas, pautas, rituales, inercias, hábitos y prácticas, formas de hacer y pensar, mentalidades y comportamientos- sedimentadas a lo largo del tiempo en forma de tradiciones, regularidades y reglas de juego no puestas en entredicho y compartidas por sus actores en el seno de las instituciones educativas" (Viñao, 2001, p. 31). Una cultura escolar que resulta difícil de cambiar.

Desde esta perspectiva comprender el peso que la "Guía del profesor" en la planificación docente de muchos profesores, y los materiales curriculares en forma de "fichas" en Educación Infantil, más allá de ciertas constataciones y críticas que chocan con la idea del docente reflexivo que construye propuestas curriculares contextualizadas, resulta crucial para comprender y vislumbrar caminos posibles de cambio real y no abocarse a la frustración cuando, con las mejores intenciones, se comienzan a desarrollar tareas 
reales de planificación y acción en las aulas incluyendo cambios notables en las dinámicas más establecidas.

Isabel ha accedido a conocer antes de las prácticas que, efectivamente, determinados usos y costumbres en relación con la planificación existen. Incluso cabe señalar que elementos están extendidos entre una parte del profesorado en ejercicio. Pero también se requiere un trabajo en mayor profundidad para atisbar las razones de tal realidad, y la complejidad de establecer otras formas de proceder, comprendiendo también que las lógicas y culturas de los formadores universitarios, de los administradores de la educación y de los docentes de Educación Infantil, y de las mismas familias de los pequeños, contienen elementos con claras diferencias. Esta reflexión cabe continuarla, después de las primeras prácticas, en el contexto de las diferentes materias teórico-prácticas, para volver a los centros en otro período, mejor preparados, incluso con el propósito de introducir innovaciones en colaboración con los propios tutores de las escuelas. En otros trabajos futuros, esperamos comentar cómo se produjeron algunos de estos procesos en relación con el caso que estamos a analizar, deteniéndonos en el desarrollo de las competencias de planificación y en la construcción de una determinada identidad profesional como profesora de Educación Infantil.

\section{Referencias}

Apple, M. (1986). Ideología y curriculum. Madrid: Akal.

Day, Ch. (2006). La pasión por enseñar. Madrid: Narcea.

Fuentes Abeledo, E. (2009). Formación de maestros y Practicum en el contexto de cambio curricular desde la perspectiva de la convergencia europea. En Raposo Rivas, M.; Martínez Figueira, M.E.; Lodeiro Enjo, L.; Fernández de la Iglesia, J.C. y Pérez Abellás, A. (Coords), El practicum más allá del empleo: Formación vs. Training (pp.103-124). Santiago de Compostela: Andavira.

Hargreaves, A. y Fullan, M. (2014). Capital profesional. Madrid: Morata.

Patton, M. (1980). Qualitative Evaluation Methods. Londres: Sage Publications.

Viñao, A. (2001). Culturas Escolares, reformas e innovaciones educativas. Con-ciencia Social: Anuario de didáctica de la geografía la historia y las Ciencias Sociales, 5, 25-46.

Simons, H. (2011). El estudio de caso: Teoría y práctica. Madrid: Morata. 